\title{
Activation of Umbilical Cord Endothelial Cells and Fetal Inflammatory Response in Preterm Infants with Chorioamnionitis and Funisitis
}

\author{
DANIELA D'ALQUEN, BORIS W. KRAMER, SILVIA SEIDENSPINNER, ALEXANDER MARX, \\ DANIEL BERG, PETER GRONECK, AND CHRISTIAN P. SPEER
}

University Children's Hospital [D.d'A., B.W.K., S.S., C.P.S.], 97080 Wuerzburg, Germany, Department of Pathology [A.M.], University of Wuerzburg, 97080 Wuerzburg, Germany, Children's Hospital of the City of Cologne [D.B., P.G.], 50735 Cologne, Germany

\begin{abstract}
Chorioamnionitis and funisitis are associated with preterm labor and postnatal morbidity. Activation of endothelium resulting in up-regulation of adhesion molecules seems to be a key mechanism in development of organ damage. We investigated whether chorioamnionitis with or without funisitis in preterm infants induced expression and shedding of adhesion molecules in the umbilical cord and resulted in increased concentrations of E-selectin, intercellular adhesion molecule (ICAM)-1, IL-1 $\beta$, IL-6, and IL-8 in the cord blood. Data were obtained by using immunohistochemistry and ELISA. Thirty-two preterm infants were divided into three groups according to histology: chorioamnionitis with funisitis, chorioamnionitis without funisitis, and controls without signs of inflammation. ICAM-1 expression on arterial endothelium was higher with funisitis compared with chorioamnionitis alone or with the control group. Similar results for ICAM-1 expression were found in venous endothelium, vascular walls, Wharton's jelly, and amnion epithelium. Endothelial E-selectin and vascular cell adhesion molecule (VCAM)-1 expression was only induced significantly with funisitis. Serum-
\end{abstract}

ABSTRACT

concentrations of soluble ICAM-1 were higher with funisitis compared with chorioamnionitis alone or control group. Similarly, concentrations of soluble E-selectin, IL-1 $\beta$, IL-6, and IL-8 were increased exclusively with funisitis. In conclusion, only chorioamnionitis with funisitis was associated with systemic inflammation and endothelial activation with up-regulation and shedding of umbilical cord adhesion molecules. We speculate that this activation of endothelium may not be limited to the umbilical cord but may also involve other organs resulting in neonatal morbidity. This underlines the importance of funisitis as a risk factor for adverse outcome. (Pediatr Res 57: 263-269, 2005)
Abbreviations
ICAM-1, intercellular adhesion molecule-1
IQR, interquartile range
PROM, premature rupture of membranes
TNF- $\alpha$, tumor necrosis factor- $\alpha$
VCAM-1, vascular cell adhesion molecule-1

Chorioamnionitis is an important risk factor for preterm delivery (1) that is associated with high perinatal morbidity (2) and mortality (3). In addition, chorioamnionitis itself is a risk factor for bronchopulmonary dysplasia, intracranial hemorrhage, and brain white matter damage (4-6).

One major pathway of infection is ascending bacteria from the vaginal tract, which can penetrate intact membranes and invade into the amnion cavity and decidua (7). This leads to infiltration of the chorioamnion by neutrophils and to chorioamnionitis resulting in high concentrations of IL- $1 \beta$, IL-6, and IL-8 in the amniotic fluid (8-10). With progression of inflammation, immune cells penetrate blood vessels and infiltrate the

Received September 24, 2003; accepted May 26, 2004.

Correspondence: Christian P. Speer, M.D., FRCPE, University Children's Hospital, Wuerzburg, Josef-Schneider-Str. 2, 97080 Wuerzburg, Germany; e-mail: speer_c@klinik.uni-wuerzburg.de

DOI: 10.1203/01.PDR.0000148713.48218.86 umbilical cord, resulting in funisitis (11). Sampson and coworkers (12) have shown by in situ hybridization that fetal cells migrate from blood vessels even through the umbilical cord tissue and reach the amniotic cavity. Therefore, funisitis is widely regarded as an inflammatory response of the fetus and has even been shown to reflect a systemic fetal inflammatory response (13-17). Furthermore, there is some evidence that funisitis is a risk factor for adverse outcome (18), chronic lung disease (19), intracranial hemorrhage (20), cerebral palsy (21), white matter damage (22), and impaired neurologic outcome (23).

Endothelial adhesion molecules play a crucial role in transmigration of leukocytes from the bloodstream to sites of inflammation. In the first step, E-selectin mediates rolling of leukocytes on the endothelium, followed by stable arrest $(9,24)$. E-selectin is a member of the selectin family and binds to sialyl Lewis $\mathrm{X}$ domain of other E-selectin molecules on 
leukocytes, predominantly neutrophils $(24,25)$. After rolling, firm adhesion of leukocytes to the endothelium, which is essential for transmigration, is mediated by VCAM- 1 and ICAM-1 (26). VCAM-1 is a member of the Ig gene superfamily (24). Its ligand is leukocyte very late antigen-4 on lymphocytes, basophils, eosinophils, monocytes, and natural killer cells, whereas VCAM-1 does not bind neutrophils (24). ICAM-1, a member of the Ig gene superfamily, binds to leukocyte function antigen-1 on all leukocytes, including neutrophils (24). The expression of these endothelial adhesion molecules can be up-regulated by inflammatory cytokines such as TNF- $\alpha$, IL-1 $\beta$, IL-6, and IL-8 (24).

We investigated whether chorioamnionitis in preterm infants in the presence or absence of funisitis induced the expression and shedding of adhesion molecules in the umbilical cord. We hypothesized that up-regulation of adhesion molecules and shedding could be part of a fetal inflammatory response syndrome with increased inflammatory mediators such as IL- $1 \beta$, IL-6, IL-8, soluble E-selectin, and soluble ICAM-1 in the fetal circulation.

\section{METHODS}

Patients. Umbilical cord blood, umbilical cord, and placenta samples from 32 preterm infants (13 female, 19 male) were taken out of a collection of 57 samples obtained from the Children's Hospital of the City of Cologne in the years 1998-2001. Median (IQR) was 26 (25-28) wk of gestation and 925 (736-1083) g for birth weight. The study was approved by the hospital ethics committee and patients' parents gave informed consent. Those patients were included in the study with the most complete set of clinical data and with sufficient amounts of cord blood sera to evaluate concentrations of IL- $1 \beta$, IL-6, IL-8, soluble E-selectin, and ICAM-1 as complete as possible. In addition, prenatal steroid prophylaxis was defined as an inclusion criterion. Hematoxylin-eosin-stained sections of placenta and umbilical cord were reviewed by a pathologist who was unaware of clinical data. Patients were assigned to three groups according to the histologic diagnose of chorioamnionitis and funisitis. Chorioamnionitis was defined as infiltration of neutrophils in the extraplacental membranes or chorionic plate (27). Funisitis was diagnosed when neutrophils infiltrated the wall of umbilical cord vessels or Wharton's jelly (27). The first group was characterized by chorioamnionitis and funisitis and will be referred to as funisitis group $(n=11)$. In the second group, only chorioamnionitis was diagnosed without inflammation of the umbilical cord, therefore, referred to as chorioamnionitis group $(n=9)$. The control group showed no inflammation of placenta and umbilical cord $(n=12)$. Characteristics of patients are shown in Table 1. There was no difference in distribution of sex, gestational age, birth weight, Apgar score after $5 \mathrm{~min}$, and umbilical cord artery $\mathrm{pH}$ between groups. Almost all mothers in all three groups had uncontrollable preterm labor with no difference between groups. The funisitis group had the highest frequency of PROM and the control group had the lowest frequency of PROM $(p<0.05$, Table 1). There was no significant difference in the frequency of PROM between the funisitis and chorioamnionitis groups and between the chorioamnionitis and control groups.

Immunohistochemistry. Immunohistochemical staining was done separately for E-selectin, VCAM-1, and ICAM-1. Serial sections were cut from formalin-fixed, paraffin-embedded umbilical cord samples and mounted onto coated slides. To improve adhesion of the tissue, slides were incubated at $37^{\circ} \mathrm{C}$ overnight. After deparaffinization and rehydration, antigen retrieval was carried out with target retrieval solution pH 6.1 (DAKO, Carpinteria, CA) using the microwave oven technique. Endogenous peroxidase activity was blocked by methyl alcohol/hydrogen peroxide, nonspecific binding by $\mathrm{PBS} / 0.02 \%$ Triton $\times 100$ and $5 \%$ BSA. Slides were incubated overnight at $4^{\circ} \mathrm{C}$ in a humidified chamber with primary monoclonal mouse antibodies in blocking solution: anti-human E-selectin (1:20, R \& D Systems, Minneapolis, MN), anti-human VCAM-1 (1:20, DAKO, Copenhagen, Denmark), anti-human ICAM-1 (1:50, Santa Cruz Biotechnology, Santa Cruz, CA). Detection was done with a secondary biotinylated anti-mouse antibody and incubation with avidin-peroxidase reagent (Vecta stain kit, Vector Laboratories, Burlingame, CA). All other reagents were obtained from Sigma Chemical Co. (St. Louis, MO), if not otherwise specified. The enzymatic reaction was developed with 3,3' diaminobenzidine and nickel sulfate in a sodium chloride acetate buffer $\mathrm{pH}$ 6.0; incubation in Tris buffer $\mathrm{pH} 7.2$ containing cobalt chloride turned the brownish color of the enzymatic product into black. After counterstaining with nuclear fast red in 5\% aluminium sulfate, samples were dehydrated and mounted.

For E-selectin and VCAM-1, the number of positive endothelial cells per vessel was counted at a magnification of $\times 40$, because we found single and clustered stained cells. ICAM-1 was expressed on all endothelial cells of all investigated vessels. Therefore, the extent of staining was measured with a four-step semiquantitative scale (magnification $\times 20$ and $\times 40$ ). Evaluation was done by two independent observers, unaware of the identity of the samples. Two to five tissue sections per slide from different regions of the umbilical cord and two arteries and one vein per tissue section were analyzed, and the average count of all results was calculated for the artery and vein of each sample.

Umbilical cord sections of a preterm infant (gestational age $25 \mathrm{wk}$ ) diagnosed with chorioamnionitis and funisitis and who died of septic shock served as positive controls for all antibodies and were included in every staining. Separate sections were also incubated with blocking solution lacking the primary antibody as a negative control.

Analysis of blood samples by ELISA. Cord blood samples were obtained immediately after birth, centrifuged, and sera were stored at $-30^{\circ} \mathrm{C}$. Soluble ICAM-1 and E-selectin concentrations were measured with commercial ELISA kits from BenderMedSystems (Vienna, Austria), with sensitivities of $3.3 \mathrm{ng} / \mathrm{mL}$ and $<0.5 \mathrm{ng} / \mathrm{mL}$, respectively. IL- $1 \beta$, IL- 6 , and IL- 8 concentrations were quantified with commercial ELISA kits from Amersham (Amersham Pharmacia Biotech Europe GmbH, Freiburg, Germany). The assay sensitivity was $0.1 \mathrm{pg} / \mathrm{mL}$ for IL- $1 \beta, 0.1 \mathrm{pg} / \mathrm{mL}$ for IL-6, and $<5 \mathrm{pg} / \mathrm{mL}$ for IL-8. Data for IL-1 $\beta$, IL- 6 , and IL- 8 were part of a publication by Tauscher et al. (28). We were not able to measure IL- 6 and IL- 8 concentrations in three samples within the chorioamnionitis group as there was not enough material available.

Statistical analysis. Differences between groups were tested with the Kruskal-Wallis $\mathrm{H}$ test (for gestational age, birth weight, umbilical cord artery $\mathrm{pH}$, and Apgar score after $5 \mathrm{~min}$ ), the $\chi^{2}$ test (for sex), the two-sided exact test by Fisher and Yates (for labor and PROM), the Mann-Whitney $U$ test (for ICAM-1 expression, VCAM-1 expression, and concentrations of inflammatory

Table 1. Characteristics of patients according the histological finding of chorioamnionitis and funisitis in placenta and umbilical cord

\begin{tabular}{|c|c|c|c|c|}
\hline \multirow[b]{2}{*}{ Gestational age (wk) } & Control $(n=12)$ & Chorioamnionitis $(n=9)$ & \multicolumn{2}{|c|}{ Funisitis $(n=11)$} \\
\hline & $26 \quad(25-28)$ & $(26-30)$ & 26 & $(25-27)$ \\
\hline Birth weight $(\mathrm{g})$ & $865 \quad(736-1000)$ & $(950-1280)$ & 860 & $(630-975)$ \\
\hline Apgar score $5 \mathrm{~min}$ & $8 \quad(7-9)$ & $(7-9)$ & 8 & $(8-8)$ \\
\hline Prenatal steroids & $12 / 12$ & $9 / 9$ & & $11 / 11$ \\
\hline Uncontrollable preterm labor & $11 / 12$ & $7 / 9$ & & $9 / 11$ \\
\hline PROM & $3 / 12$ & $6 / 9$ & & $8 / 11$ \\
\hline
\end{tabular}

There was no significant difference in gestational age, birth weight, Apgar score after $5 \mathrm{~min}$, umbilical cord artery pH, and uncontrollable preterm labor between groups. The control group had the lowest incidence of PROM, the funisitis group the highest incidence ( $p<0.05$; two-sided exact test by Fisher and Yates). Umbilical cord artery $\mathrm{pH}$ was not available for one patient in the funisitis group and for one patient in the control group. Results are presented as median (IQR). 
mediators), the Kendall tau-correlation test (for correlation between concentrations of inflammatory mediators in the cord blood and expression of adhesion molecules in the umbilical cord), and the two-factor-variant-analysis of Puri and Sen (for the difference between arterial and venous ICAM-1 expression). A value of $p \leq 0.05$ was considered significant.

\section{RESULTS}

\section{Expression of Adhesion Molecules in the Umbilical Cord}

E-selectin. E-selectin expression was detected in only 3 out of 32 cases, and expression was restricted to endothelial cells. The staining pattern consisted of isolated and clustered positive cells (Fig. 1). Arterial endothelium was positive in three cases; two of these also showed expression on venous endothelium. All positive cases belonged to the funisitis group, whereas there was no E-selectin expression in the other groups (data not shown).

VCAM-1. Similar to E-selectin, VCAM-1 was exclusively detected on endothelial cells. Positive cells were mostly found in clusters or as isolated cells (Fig. 2). Thirteen out of 32 cases showed positive VCAM-1 staining of arterial endothelium, most of them belonging to the funisitis group, with 8 positive cases out of 11 (Table 2). With chorioamnionitis alone, only 4 out of 9 cases revealed arterial VCAM-1 expression, whereas in the control group only 1 out of 12 cases was positive for VCAM-1. Comparison of numbers of positive cells per artery showed a higher expression of VCAM-1 in the funisitis group compared with the chorioamnionitis and control groups $(p<$ 0.05 and $p<0.005$, respectively). There was no difference between the chorioamnionitis and control groups.

VCAM-1 expression on venous endothelium was less frequent when compared with expression on arterial endothelium with 8 out of 32 samples (Table 2). With funisitis, 6 out of 11 cases revealed VCAM-1 expression on venous endothelium, whereas there were only 2 positive cases out of 9 with chorioamnionitis alone and none in the control group. The number of positive stained cells per vein were higher in the funisitis group compared with the control group $(p<0.005)$, whereas there was no difference between the chorioamnionitis group and the two other groups.

ICAM-1. ICAM-1 was expressed on all endothelial cells of all investigated vessels. In addition, it could be detected in vascular wall (Fig. 3), Wharton's jelly, and amnion epithelium (data not shown). Staining intensity was measured with a four-step semiquantitative scale ranging from 1 to 4 for endothelium and from 0 to 3 for vascular wall (Fig. 3), Wharton's jelly, and amnion epithelium. In all compartments of the umbilical cord, ICAM-1 expression was higher within the funisitis group compared with chorioamnionitis alone or with the control group, with no difference between the chorioamnionitis and the control groups. ICAM-1 expression intensity on arterial endothelium raised from $2.0(2.0-2.4)$ in the control group and $2.1(1.8-2.4)$ in the chorioamnionitis group to 2.5 $(2.4-2.8)$ in the funisitis group $(p<0.005$ and $p<0.001$, respectively; Fig. 4A). ICAM-1 expression in arterial vascular walls increased from $0.7(0.5-0.9)$ in the control group and 0.9 $(0.4-0.9)$ with chorioamnionitis alone to $2.3(1.8-2.5)$ with funisitis $(p<0.005$ and $p<0.005$, respectively; Fig. $4 B)$. On venous endothelium, ICAM-1 expression raised from $1.3(1.1-$ $1.7)$ in the control group and $1.4(1.3-1.5)$ with chorioamnionitis alone to $2.3(2.2-2.7)$ with funisitis $(p<0.0005$ and $p<$ 0.0001 , respectively; Fig. $4 C$ ). ICAM-1 staining intensity in venous vascular walls increased from $1.0(0.7-1.0)$ in the control group and $0.7(0.5-1.0)$ in the chorioamnionitis group to $2.0(1.9-2.3)$ in the funisitis group $(p<0.0001$ and $p<$ 0.0001, respectively; Fig. 4D). Similarly, ICAM-1 expression in the Wharton's jelly and amnion epithelium increased significantly in the funisitis group compared with the other groups (data not shown).

In the chorioamnionitis and control group, ICAM-1 expression on venous endothelium was lower than on arterial endothelium [vein, $1.4(1.3-1.5)$ versus artery, $2.1(1.8-2.4)$, and vein, 1.3 (1.1-1.7) versus artery, 2.0 (2.0-2.4), respectively, $p<0.05$; Fig. 4, $A$ and $C$ ]. In the funisitis group, however, ICAM-1 expression on venous endothelium raised to the same level as on arterial endothelium [vein, 2.3 (2.2-2.7) versus artery, 2.5 (2.4-2.8); Fig. 4, $A$ and $C$ ].

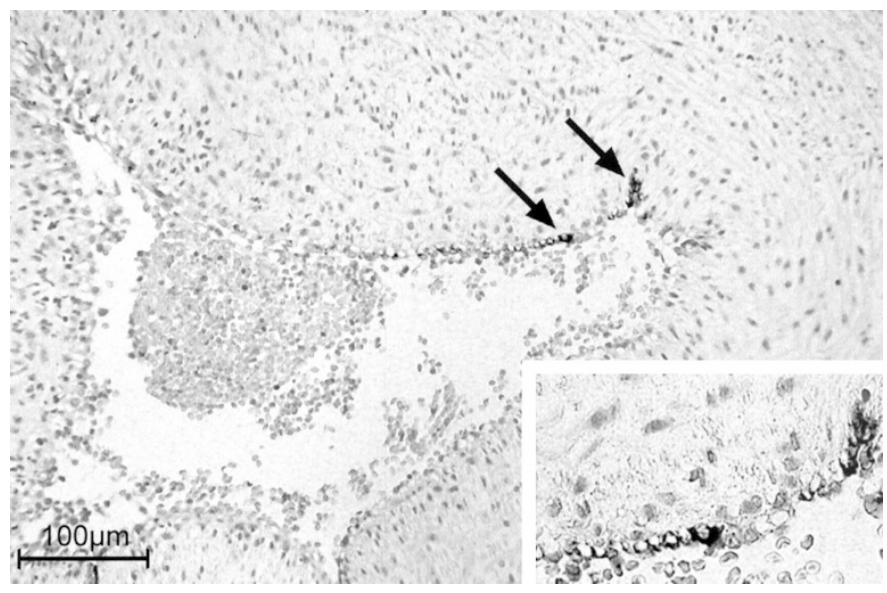

Figure 1. Immunohistochemical staining of E-selectin $(\rightarrow)$ on umbilical artery endothelium of a 26 -wk preterm infant with funisitis. Magnification $\times 20$ (inset, $\times 40$ ).

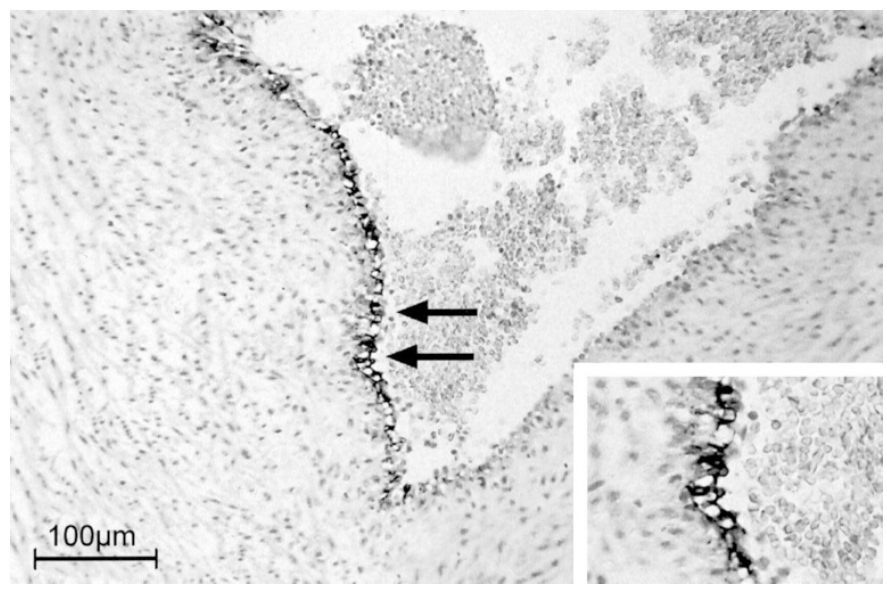

Figure 2. Immunohistochemical staining of VCAM-1 $(\rightarrow)$ on umbilical artery endothelium of a 26-wk preterm infant with funisitis. Magnification $\times 20$ (inset, $\times 40$ ). 
Table 2. Immunohistochemical detection of VCAM-1 on endothelium of umbilical cord artery and vein

\begin{tabular}{lccc}
\hline & $\begin{array}{c}\text { Control } \\
(n=12)\end{array}$ & $\begin{array}{c}\text { Chorioamnionitis } \\
(n=9)\end{array}$ & $\begin{array}{c}\text { Funisitis } \\
(n=11)\end{array}$ \\
\hline VCAM-1 artery & $1 / 12$ & $4 / 9$ & $8 / 11$ \\
VCAM-1 vein & $0 / 12$ & $2 / 9$ & $6 / 11$ \\
Positive cells artery (average count/sample), group median & $0(0-0)^{* *}$ & $0(0-0.3)^{*}$ & $1.3(0.1-12.1)^{*}, * *$ \\
Range & 0.5 & $0.3-0.4$ & $0.2-20.3$ \\
Positive cells vein (average count/sample), group median & $0(0-0) \dagger$ & $0(0-0)$ & $0.06(0-0.8) \dagger$ \\
Range & 0 & $0.3-0.6$ & $0.06-4.6$ \\
\hline
\end{tabular}

Values of VCAM-1 artery and VCAM-1 vein are expressed as number of positive samples out of the total number per group. For each sample, an average count for positive arterial and venous endothelial cells was calculated out of all analyzed tissue sections and vessels. Then the median was calculated for each group. Arterial VCAM-1 expression was higher in the funisitis group compared with the chorioamnionitis group $(* p<0.05)$ or control group $(* * p<0.005)$. For venous VCAM-1 expression, only comparison of the funisitis group with the control group showed a significant increased VCAM- 1 expression $(\dagger p<0.005$; Mann-Whitney $U$ test). Results are presented as median (IQR).

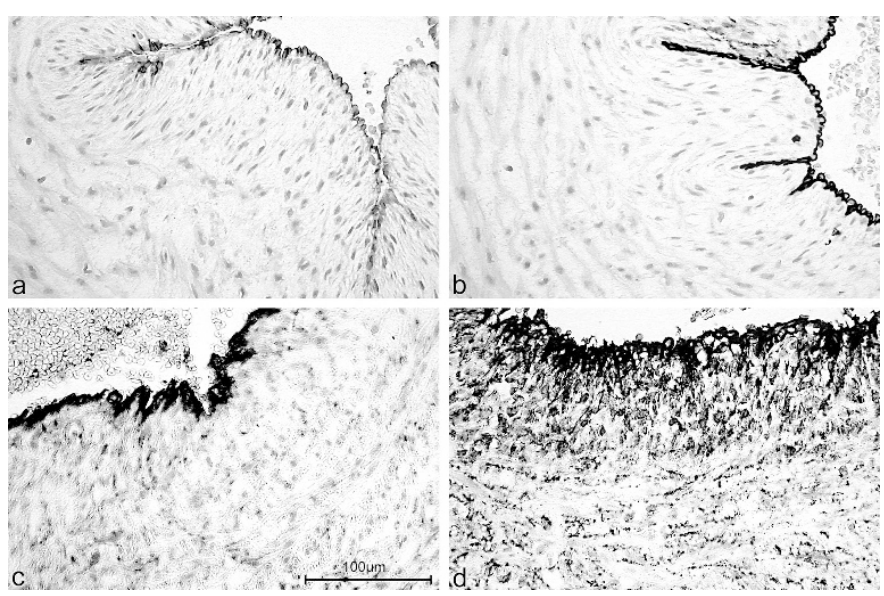

Figure 3. Semiquantitative grading of immunohistochemical ICAM-1 staining: grade $1-4$ for endothelium ( $a-d$, arterial endothelium shown here), grade $0-3$ for vascular wall $(a-d)$. Magnification $\times 40$.

\section{Cytokines and Soluble Adhesion Molecules in the Cord Blood}

Concentrations of all investigated cytokines and soluble adhesion molecules were higher with funisitis compared with chorioamnionitis alone or with the control group, with no difference between the chorioamnionitis and control group. The median concentration of soluble E-selectin in the funisitis group showed a 1.5 -fold increase compared with the chorioamnionitis group $(p=0.05)$ and a 3.5 -fold increase compared with the control group $(p<0.005$; Fig. $5 A)$. The median concentration of soluble ICAM-1 in the funisitis group increased 2 times compared with the chorioamnionitis group ( $p$ $<0.005)$ and with the control group $(p<0.001$; Fig. $5 B)$. The median concentration of IL- 6 in the funisitis group revealed a 60 -fold increase compared with the chorioamnionitis group ( $p$ $<0.005)$ and an 80-fold increase compared with the control group ( $p<0.0005$; Fig. $5 C$ ). The median concentration of IL- $1 \beta$ in the funisitis group increased 5 times compared with the chorioamnionitis group $(p<0.05)$ and 13.3 times compared with the control group ( $p<0.005$; data not shown). The median concentration of IL-8 in the funisitis group showed a 6.7 -fold increase compared with the chorioamnionitis group ( $p$ $<0.005)$ and an 8.6-fold increase compared with the control group ( $p<0.001$; data not shown).
There was a direct correlation between concentrations of soluble E-selectin in the cord blood and the immunohistochemical expression of ICAM-1 on arterial and venous endothelium, in arterial and venous vascular walls, and in the Wharton's jelly of the umbilical cord (tau $<0.05$, data not shown). There was no correlation between soluble E-selectin concentrations and expression of E-selectin on endothelium of umbilical cord vessels, which was positive in only three cases (data not shown). Concentrations of soluble ICAM-1, IL-6, and IL8 in the cord blood correlated with the expression of E-selectin, VCAM-1, and ICAM-1 in all compartments of the umbilical cord (tau $<0.01$, tau $<0.01$, and tau $<0.05$, respectively, data not shown). In addition, there was a correlation between concentrations of cord blood IL-1 $\beta$ and expression of Eselectin and VCAM-1 on arterial and venous endothelium and ICAM-1 on venous endothelium, in arterial and venous vascular walls, in the Wharton's jelly, and the amnion epithelium of the umbilical cord (tau $<0.05$, data not shown).

\section{DISCUSSION}

In the present study, we investigated for the first time the expression of adhesion molecules in the umbilical cord together with concentrations of soluble adhesion molecules and proinflammatory cytokines in the cord blood in preterm infants with chorioamnionitis or funisitis. In our study, only chorioamnionitis with funisitis but not chorioamnionitis alone resulted in up-regulation of adhesion molecules in the umbilical cord. This up-regulation seems to be part of a systemic inflammatory response as concentrations of soluble adhesion molecules and cytokines increased only in the funisitis group.

Several factors may influence the expression of adhesion molecules, such as gestational age $(29,30)$. Therefore, it was important that there was no difference in distribution of gestational age between groups in our study.

E-selectin expression was positive in only 3 out of 32 cases, all belonging to the funisitis group. Expression was confined to the endothelium of umbilical cord vessels as reported previously (24). The reasons for the rare detection of positive E-selectin staining in our samples remain elusive. Several in vivo and in vitro investigations have shown an inhibitory effect of steroids on the expression of all three adhesion molecules $(24,31,32)$. In our study, however, all mothers had received antenatal steroids to induce fetal lung maturation, so there was 

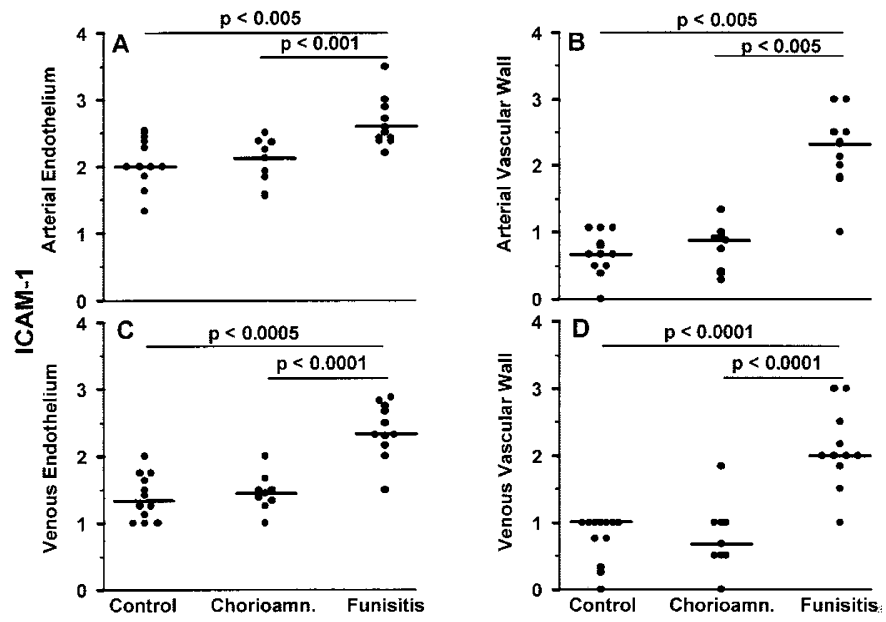

Figure 4. Comparison of ICAM-1 expression between funisitis, chorioamnionitis, and control group. (A) arterial endothelium, $(B)$ arterial vascular wall, $(C)$ venous endothelium, $(D)$ venous vascular wall. Filled circle represents a single sample, - represents the median. Comparison between groups was done with Mann-Whitney $U$ test.

no difference in exposure to steroids between the groups. Despite the influence of steroids on all three adhesion molecules, we found a more frequent expression of VCAM-1 and ICAM-1 compared with E-selectin. Therefore, it seems unlikely that steroids alone are responsible for the low incidence of E-selectin positive staining. Secondly, E-selectin seems not to be expressed constitutively on endothelium (33). In a recent study, none of the preterm infants without exposure to intraamniotic infection had E-selectin expression in the umbilical cord (34). This is consistent with our findings that there was no E-selectin expression in the control group. As shown in in vitro models, inflammatory mediators such as TNF- $\alpha$ and IL- $1 \beta$ induced E-selectin expression on endothelial cells, but kinetic studies showed a transient induction with a peak at 2-6 h and a rapid decline to basal levels at $24 \mathrm{~h}$ after stimulation $(35,36)$. Because of the short time period of up-regulation, we might not have been able to detect E-selectin expression in the majority of cases.

We hypothesized that low E-selectin levels on the endothelium might be due to shedding into the circulation. In fact, increased levels of soluble E-selectin were only found in patients exposed to funisitis. There was no difference in con- centrations between the chorioamnionitis and control group. This suggests that endothelial expression of E-selectin in the funisitis group is reflected by enhanced shedding. We were able to show that even very immature infants were able to respond to an inflammatory stimulus with a marked increase of soluble adhesion molecules. In contrast, Austgulen and colleagues (29) could only detect increased soluble ICAM-1 and E-selectin levels in term but not in preterm infants with signs of inflammation. In their study, however, inflammation group was defined merely by means of clinical data and not by histologic evaluation.

Similar to E-selectin, VCAM-1 could exclusively be detected on endothelial cells of umbilical cord vessels. We were able to detect VCAM-1 in 13 out of 32 cases but only in 1 out of 12 cases in the control group. In accordance with our findings, VCAM-1 could not be detected on endothelium of umbilical cord vessels in 61 preterm infants without intraamniotic inflammation (34). These data indicate that VCAM-1 is not constitutively expressed at detectable levels on endothelium but can be up-regulated upon inflammation $(33,34,37)$. Compared with E-selectin, VCAM-1 expression is sustained longer with a peak at $12-18 \mathrm{~h}$ after stimulation $(9,24)$, which might be a possible reason for the higher frequency of VCAM1 -positive samples in our study.

In contrast to E-selectin and VCAM-1, ICAM-1 could be detected on endothelium as well as in vascular walls, Wharton's jelly, and amnion epithelium. Expression in vascular walls could be due to expression on smooth muscle cells themselves, as shown in several in vitro experiments $(38,39)$, or could be a result of positive stained macrophages, neutrophils, or T-lymphocytes $(9,24)$. So far, there is no available data on ICAM-1 expression in Wharton's jelly, but we assume that positive staining is at least partly due to staining of inflammatory cells. The ability of amnion cells to express ICAM-1 has previously been shown in vitro (40), but not in vivo as in our study. In contrast to E-selectin and VCAM-1, endothelial ICAM-1 expression comprised the whole circumference of all investigated vessels, including the control group. This constitutive low-level expression has been shown to be up-regulated by a variety of cytokines (IL-1, IL-6, IL-8, TNF- $\alpha$ ) with a maximum 9-24 $\mathrm{h}$ after stimulation and a persistently high expression $(9,24)$. In our study, ICAM-1
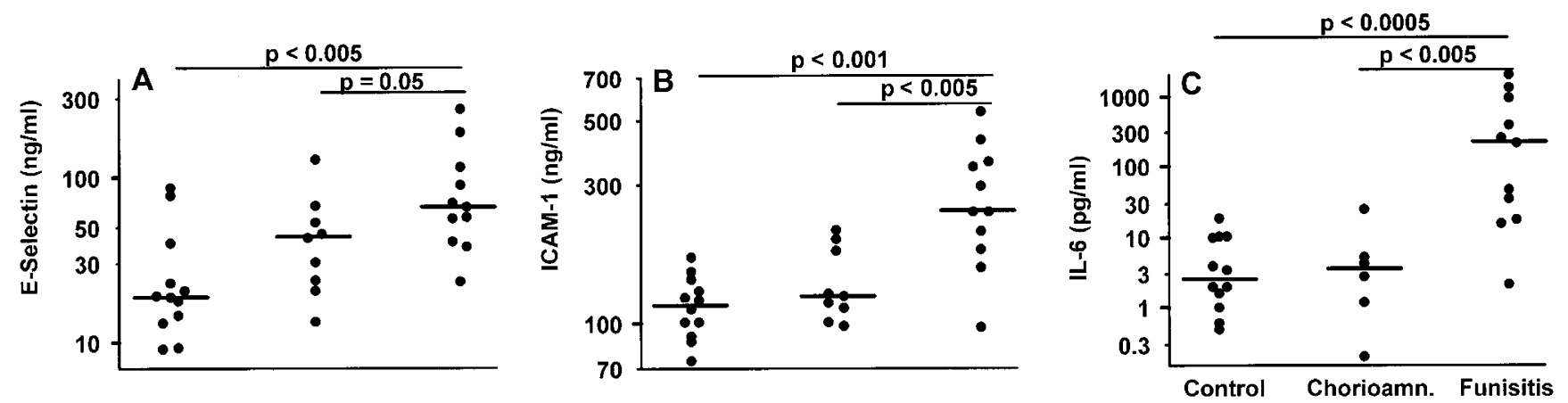

Figure 5. Comparison of soluble E-selectin $(A)$, soluble ICAM-1 $(B)$, and IL-6 $(C)$ concentrations in the cord blood between funisitis, chorioamnionitis, and control group (logarithmic scale on $y$ axis). Filled circle represents a single sample, - represents the median. Comparison between groups was done with Mann-Whitney $U$ test. 
expression increased significantly in all compartments of the umbilical cord with funisitis compared with chorioamnionitis alone or the control group.

Besides inflammation, ICAM-1 expression has been shown to be up-regulated by the onset of labor (34). Therefore, it was important that there were no differences in the onset of labor between our groups. Steinborn and co-workers (34) were able to demonstrate that ICAM-1 was up-regulated exclusively on endothelium of umbilical cord vessels with the onset of labor and expression was stronger on arterial than on venous endothelium in noninfected pregnancies. However, in our study design, we found that funisitis led to an up-regulation of ICAM-1 in all compartments of the umbilical cord and ICAM-1 expression raised to the same level on venous as on arterial endothelium, whereas ICAM-1 expression in the chorioamnionitis and control group was stronger on arterial than on venous endothelium. Furthermore, in our study, upregulation of adhesion molecules in preterm infants with funisitis resulted in enhanced shedding with high concentrations of soluble E-selectin and ICAM-1 in the circulation, whereas labor had no effect on soluble ICAM-1 levels (41). Therefore, labor-associated up-regulation of ICAM-1 seems to differ from inflammation-associated up-regulation in the expression pattern and kinetics of shedding.

The reason for the higher expression of ICAM-1 on arterial compared with venous endothelium, which was described by Steinborn and co-workers (34) and confirmed by us remains unclear. One may speculate that differences in flow velocity and shear stress in arteries compared with veins may result in different expression of adhesion molecules. An impaired flow was recently shown to affect the adhesion of inflammatory cells to endothelial cells and their expression of adhesion molecules (42).

To evaluate whether up-regulation of adhesion molecules in funisitis was a local phenomenon or part of a fetal systemic inflammatory response, we investigated levels of IL- $1 \beta$, IL-6, and IL-8 in the cord blood. In parallel to up-regulation of adhesion molecules, those cytokines were strongly increased in funisitis compared with chorioamnionitis alone or the control group. No difference in cytokine concentrations between the chorioamnionitis group and the control group could be detected. In contrast, several studies have shown an increase in IL-1 $\beta$, IL-6, and IL-8 levels in the fetal circulation in case of chorioamnionitis $(6,43,44)$, associated with an increased risk for intracranial hemorrhage (28), but the chorioamnionitis group in these studies was not subdivided according to the presence of funisitis. Supporting our findings, other studies showed increased fetal blood IL-6 concentrations in the presence of funisitis $(13,15,16,45)$. In addition, IL- 6 has been shown to be a useful marker for early neonatal sepsis $(46,47)$.

We may conclude that up-regulation of endothelial adhesion molecules in chorioamnionitis with funisitis represents a systemic fetal inflammatory response with enhanced concentrations of inflammatory mediators in the cord blood. In contrast, we could not find an up-regulation of umbilical cord endothelial adhesion molecules or signs of a fetal systemic inflammatory response in our study group with chorioamnionitis alone.
Therefore, we speculate that this represents a localized inflammatory process, confined mainly to the maternal compartment.

Cytokines themselves may cause cell damage (17), but in addition, they may induce up-regulation and shedding of adhesion molecules as we found high concentrations of soluble E-selectin and ICAM-1 in the circulation in funisitis compared with chorioamnionitis alone or the control group. In vivo studies investigating concentrations of soluble adhesion molecules assume that high levels reflect high expression on endothelium, but immunohistochemical expression was not investigated $(48,49)$. In our study, we were able to show that enhanced expression of ICAM-1 and E-selectin in funisitis was accompanied by higher concentrations of the soluble forms in the circulation in that group. Controversial data exist concerning the association between blood concentrations of soluble adhesion molecules and the onset of neonatal infection and sepsis $(46,47,50,51)$.

These soluble adhesion molecules might have a systemic effect on the infant with possible damage of other organs. It has been suggested that elevated levels of soluble E-selectin and ICAM-1 in the circulation of preterm infants might be a risk factor for bronchopulmonary dysplasia $(52,53)$. It still remains unclear whether there is a direct pathophysiological effect of soluble adhesion molecules that causes organ damage (54) or whether it is only the up-regulation of adhesion molecules on endothelium with a subsequent influx of inflammatory cells and increased expression of proinflammatory mediators (55) that is responsible for tissue injury. Up-regulation of adhesion molecules on endothelial cells and binding of leukocytes induces activation of endothelial cells resulting in transmigration of leukocytes and tissue damage. In addition, activation of the coagulatory cascade with thrombosis or bleeding can take place $(56,57)$. Supporting our hypothesis, funisitis has been reported to reflect a systemic fetal inflammatory response $(16,17)$ and to be a risk factor for adverse outcome (18), intracranial hemorrhage (20), and impaired neurologic outcome with cerebral palsy/white matter damage (17,21-23), whereas there was no association between chorioamnionitis alone and cerebral palsy after adjusting the analysis for gestational age at birth $(17,21)$.

Taken together, our results indicate that chorioamnionitis with funisitis induces up-regulation of adhesion molecules in the umbilical cord. This up-regulation seems to be part of a fetal inflammatory response syndrome with increased concentrations of cytokines and shedded soluble adhesion molecules in the fetal circulation (58). Therefore, it is likely that activation of endothelium is not limited to the umbilical cord but can involve other fetal organs, possibly leading to damage and predicting adverse outcome in the case of funisitis.

\section{REFERENCES}

1. Goldenberg RL, Andrews WW, Hauth JC 2002 Choriodecidual infection and preterm birth. Nutr Rev 60:S19-S25

2. Morrison JC 1990 Preterm birth: a puzzle worth solving. Obstet Gynecol 76:5S-12S

3. Copper RL, Goldenberg RL, Creasy RK, DuBard MB, Davis RO, Entman SS, Iams JD, Cliver SP 1993 A multicenter study of preterm birth weight and gestational age-specific neonatal mortality. Am J Obstet Gynecol 168:78-84

4. Speer CP 2003 Inflammation and bronchopulmonary dysplasia. Semin Neonatol $8: 29-38$ 
5. Bracci R, Buonocore G 2003 Chorioamnionitis: a risk factor for fetal and neonatal morbidity. Biol Neonate 83:85-96

6. Gomez R, Romero R, Ghezzi F, Yoon BH, Mazor M, Berry SM 1998 The fetal inflammatory response syndrome. Am J Obstet Gynecol 179:194-202

7. Toti P, De Felice C 2001 Chorioamnionitis and fetal/neonatal brain injury. Bio Neonate 79:201-204

8. Yoon BH, Romero R, Kim CJ, Jun JK, Gomez R, Choi JH, Syn HC 1995 Amniotic fluid interleukin-6: a sensitive test for antenatal diagnosis of acute inflammatory lesions of preterm placenta and prediction of perinatal morbidity. Am J Obste Gynecol 172:960-970

9. Springer TA 1990 Adhesion receptors of the immune system. Nature 346:425-434

10. Romero R, Mazor M, Brandt F, Sepulveda W, Avila C, Cotton DB, Dinarello CA 1992 Interleukin-1 alpha and interleukin-1 beta in preterm and term human parturition. Am J Reprod Immunol 27:117-123

11. Romero R, Mazor M 1988 Infection and preterm labor. Clin Obstet Gynecol 31:553-584

12. Sampson JE, Theve RP, Blatman RN, Shipp TD, Bianchi DW, Ward BE, Jack RM 1997 Fetal origin of amniotic fluid polymorphonuclear leukocytes. Am J Obste Gynecol 176:77-81

13. Pacora P, Chaiworapongsa T, Maymon E, Kim YM, Gomez R, Yoon BH, Ghezzi F, Berry SM, Qureshi F, Jacques SM, Kim JC, Kadar N, Romero R 2002 Funisitis an chorionic vasculitis: the histological counterpart of the fetal inflammatory response syndrome. J Matern Fetal Neonatal Med 11:18-25

14. Maudsley RF, Brix GA, Hinton NA, Robertson EM, Bryans AM, Haust MD 1966 Placental inflammation and infection. A prospective bacteriologic and histologic study. Am J Obstet Gynecol 95:648-659

15. Naccasha N, Hinson R, Montag A, Ismail M, Bentz L, Mittendorf R 2001 Association between funisitis and elevated interleukin-6 in cord blood. Obstet Gynecol 97:220 224

16. Yoon BH, Romero R, Park JS, Kim M, Oh SY, Kim CJ, Jun JK 2000 The relationship among inflammatory lesions of the umbilical cord (funisitis), umbilical cord plasm interleukin 6 concentration, amniotic fluid infection, and neonatal sepsis. Am J Obstet Gynecol 183:1124-1129

17. Yoon BH, Park CW, Chaiworapongsa T 2003 Intrauterine infection and the development of cerebral palsy. BJOG 10:124-127

18. Kim CJ, Yoon BH, Romero R, Moon JB, Kim M, Park SS, Chi JG 2001 Umbilica arteritis and phlebitis mark different stages of the fetal inflammatory response. Am J Obstet Gynecol 185:496-500

19. Matsuda T, Nakajima T, Hattori S, Hanatani K, Fukazawa Y, Kobayashi K, Fujimoto S 1997 Necrotizing funisitis: clinical significance and association with chronic lung disease in premature infants. Am J Obstet Gynecol 177:1402-1407

20. DiSalvo D 1998 The correlation between placental pathology and intraventricula hemorrhage in the preterm infant. The Developmental Epidemiology Network Investigators. Pediatr Res 43:15-19

21. Yoon BH, Romero R, Park JS, Kim CJ, Kim SH, Choi JH, Han TR 2000 Fetal exposure to an intra-amniotic inflammation and the development of cerebral palsy at the age of three years. Am J Obstet Gynecol 182:675-681

22. Leviton A, Paneth N, Reuss ML, Susser M, Allred EN, Dammann O, Kuban K, Van Marter LJ, Pagano M, Hegyi T, Hiatt M, Sanocka U, Shahrivar F, Abiri M, Disalvo D, Doubilet P, Kairam R, Kazam E, Kirpekar M, Rosenfeld D, Schonfeld S, Share J, Collins M, Genest D, Shen-Schwarz S, et al 1999 Maternal infection, fetal inflammatory response, and brain damage in very low birth weight infants. Developmenta Epidemiology Network Investigators. Pediatr Res 46:566-575

23. Mittendorf R, Montag AG, MacMillan W, Janeczek S, Pryde PG, Besinger RE, Gianopoulos JG, Roizen N 2003 Components of the systemic fetal inflammatory response syndrome as predictors of impaired neurologic outcomes in children. Am J Obstet Gynecol 188:1438-1444; discussion 1444-1446

24. Radi ZA, Kehrli ME Jr, Ackermann MR 2001 Cell adhesion molecules, leukocyte trafficking, and strategies to reduce leukocyte infiltration. J Vet Intern Med 15:516529

25. Phillips ML, Nudelman E, Gaeta FC, Perez M, Singhal AK, Hakomori S, Paulson JC 1990 ELAM-1 mediates cell adhesion by recognition of a carbohydrate ligand, sialyl-Lex. Science 250:1130-1132

26. Albelda SM, Smith CW, Ward PA 1994 Adhesion molecules and inflammatory injury. FASEB J 8:504-512

27. Salafia CM, Weigl C, Silberman L 1989 The prevalence and distribution of acute placental inflammation in uncomplicated term pregnancies. Obstet Gynecol 73:383389

28. Tauscher MK, Berg D, Brockmann M, Seidenspinner S, Speer CP, Groneck P 2003 Association of histologic chorioamnionitis, increased levels of cord blood cytokines, and intracerebral hemorrhage in preterm neonates. Biol Neonate 83:166-170

29. Austgulen R, Arntzen KJ, Haereid PE, Aag S, Dollner H 1997 Infections in neonates delivered at term are associated with increased serum levels of ICAM-1 and Eselectin. Acta Paediatr 86:274-280

30. Dogan A, MacDonald TT, Spencer J 1993 Ontogeny and induction of adhesion molecule expression in human fetal intestine. Clin Exp Immunol 91:532-537

31. Aziz KE, Wakefield D 1996 Modulation of endothelial cell expression of ICAM-1, E-selectin, and VCAM-1 by beta-estradiol, progesterone, and dexamethasone. Cell Immunol 167:79-85
32. Chen W, Lee JY, Hsieh WC 2002 Effects of dexamethasone and sex hormones on cytokine-induced cellular adhesion molecule expression in human endothelial cells. Eur J Dermatol 12:445-448

33. Vanhee D, Delneste Y, Lassalle P, Gosset P, Joseph M, Tonnel AB 1994 Modulation of endothelial cell adhesion molecule expression in a situation of chronic inflammatory stimulation. Cell Immunol 155:446-456

34. Steinborn A, Sohn C, Heger S, Niederhut A, Hildenbrand R, Kaufmann M 1999 Labour-associated expression of intercellular adhesion molecule-1 (ICAM- 1) in placental endothelial cells indicates participation of immunological processes in parturition. Placenta 20:567-573

35. Scholz D, Devaux B, Hirche A, Potzsch B, Kropp B, Schaper W, Schaper J 1996 Expression of adhesion molecules is specific and time-dependent in cytokinestimulated endothelial cells in culture. Cell Tissue Res 284:415-423

36. Bevilacqua MP, Stengelin S, Gimbrone MA Jr, Seed B 1989 Endothelial leukocyte adhesion molecule 1: an inducible receptor for neutrophils related to complement regulatory proteins and lectins. Science 243:1160-1165

37. Craven CM, Ward K 2000 Fetal endothelial cells express vascular cell adhesion molecule in the setting of chorioamnionitis. Am J Reprod Immunol 43:259-263

38. Wang X, Feuerstein GZ, Gu JL, Lysko PG, Yue TL 1995 Interleukin-1 beta induces expression of adhesion molecules in human vascular smooth muscle cells and enhances adhesion of leukocytes to smooth muscle cells. Atherosclerosis 115:89-98

39. Braun M, Pietsch P, Schror K, Baumann G, Felix SB 1999 Cellular adhesion molecules on vascular smooth muscle cells. Cardiovasc Res 41:395-401

40. Marvin KW, Hansen WR, Miller HC, Eykholt RL, Mitchell MD 1999 Amnionderived cells express intercellular adhesion molecule-1: regulation by cytokines. J Mol Endocrinol 22:193-205

41. Hata T, Kawamura T, Inada K, Fujiwaki R, Ariyuki Y, Hata K, Kitao M 1996 Cord blood cytokines and soluble adhesion molecules in vaginal and cesarean delivered neonates. Gynecol Obstet Invest 42:102-104

42. Chiu JJ, Chen CN, Lee PL, Yang CT, Chuang HS, Chien S, Usami S 2003 Analysis of the effect of disturbed flow on monocytic adhesion to endothelial cells. J Biomech 36:1883-1895

43. Shimoya K, Matsuzaki N, Taniguchi T, Jo T, Saji F, Kitajima H, Fujimura M, Nakayama M, Tanizawa O 1992 Interleukin-8 in cord sera: a sensitive and specific marker for the detection of preterm chorioamnionitis. J Infect Dis 165:957-960

44. Singh B, Merchant P, Walker CR, Kryworuchko M, Diaz-Mitoma F 1996 Interleukin-6 expression in cord blood of patients with clinical chorioamnionitis. Pediatr Res 39:976-979

45. Rogers BB, Alexander JM, Head J, McIntire D, Leveno KJ 2002 Umbilical vein interleukin-6 levels correlate with the severity of placental inflammation and gestational age. Hum Pathol 33:335-340

46. Lehrnbecher T, Schrod L, Rutsch P, Roos T, Martius J, von Stockhausen HB 1996 Immunologic parameters in cord blood indicating early-onset sepsis. Biol Neonate 70:206-212

47. Smulian JC, Bhandari V, Campbell WA, Rodis JF, Vintzileos AM 1997 Value of umbilical artery and vein levels of interleukin-6 and soluble intracellular adhesion molecule- 1 as predictors of neonatal hematologic indices and suspected early sepsis. J Matern Fetal Med 6:254-259

48. Boldt J, Wollbruck M, Kuhn D, Linke LC, Hempelmann G 1995 Do plasma levels of circulating soluble adhesion molecules differ between surviving and nonsurviving critically ill patients? Chest 107:787-792

49. Law MM, Cryer HG, Abraham E 1994 Elevated levels of soluble ICAM-1 correlate with the development of multiple organ failure in severely injured trauma patients. J Trauma 37:100-109; discussion 109-110

50. Hansen AB, Verder H, Staun-Olsen P 2000 Soluble intercellular adhesion molecule and C-reactive protein as early markers of infection in newborns. J Perinat Med 28:97-103

51. Darai E, Poncelet C, Soriano D, Lejeune V, Bringuier AF, Madelenat P, Feldmann G, Scoazec JY 2002 Soluble intercellular adhesion molecule 1 in umbilical cord serum: potential for the diagnosis of neonatal infections. Fetal Diagn Ther 17:167-172

52. Ramsay PL, O'Brian Smith E, Hegemier S, Welty SE 1998 Early clinical markers for the development of bronchopulmonary dysplasia: soluble E-Selectin and ICAM-1. Pediatrics 102:927-932

53. Ballabh P, Kumari J, Krauss AN, Shin JJ, Jain A, Auld PA, Lesser ML, CunninghamRundles S 2003 Soluble e-selectin, soluble L-selectin and soluble icam-1 in bronchopulmonary dysplasia, and changes with dexamethasone. Pediatrics 111:461-468

54. Pleines UE, Stover JF, Kossmann T, Trentz O, Morganti-Kossmann MC 1998 Soluble ICAM-1 in CSF coincides with the extent of cerebral damage in patients with severe traumatic brain injury. J Neurotrauma 15:399-409

55. Schmidt B, Cao L, Mackensen-Haen S, Kendziorra H, Klingel K, Speer CP 2001 Chorioamnionitis and inflammation of the fetal lung. Am J Obstet Gynecol 185:173177

56. Dammann O, Leviton A 1997 Maternal intrauterine infection, cytokines, and brain damage in the preterm newborn. Pediatr Res 42:1-8

57. Blann AD 2000 Endothelial cell activation, injury, damage and dysfunction: separate entities or mutual terms? Blood Coagul Fibrinolysis 11:623-630

58. Speer CP 2001 New insights into the pathogenesis of pulmonary inflammation in preterm infants. Biol Neonate 79:205-209 